\title{
Improvement of Lipids and Reduction of Oxidative Stress With Octacosanol After Taekwondo Training
}

\author{
Sang-Ho Lee, Steven D. Scott, Elizabeth J. Pekas, Jeong-Gi Lee, and Song-Young Park
}

\begin{abstract}
Purpose: Athletes in combat sports undergo rapid changes in body weight prior to competition in order to gain a size advantage over their opponent. However, these large weight changes with concomitant high-intensity exercise training create poor lipid profiles and high levels of oxidative stress, which can be detrimental to health and sport performance. Therefore, the purpose of this study was to investigate the ability of the nutritional supplement octacosanol to combat the physiological detriments that occur in taekwondo players during rapid weight loss with high-intensity exercise training. Methods: A total of 26 male taekwondo players were randomly divided into 2 groups: An experimental group performed a 5\% weight-loss and taekwondo training program with 40-mg octacosanol intake $(\mathrm{OCT} ; \mathrm{n}=13)$ for $6 \mathrm{~d}$, and a control group performed the same weight-loss and taekwondo training program with a placebo $(\mathrm{CON} ; \mathrm{n}=13)$. Results: There were significant $(P<.05)$ group $\times$ time interactions for low-density lipoprotein and triglycerides, which significantly decreased $(\Delta 18$ [5] mg/dL and $\Delta 80$ [7] $\mathrm{mg} / \mathrm{dL}$, respectively), and high-density lipoprotein, which significantly increased $(\Delta 10[7] \mathrm{mg} / \mathrm{dL})$, in the OCT group compared with the CON group. There were also significant $(P<.05)$ group $\times$ time interactions for superoxide dismutase (SOD), glutathione peroxidase $(\mathrm{GPx})$, and malondialdehyde (MDA), with SOD increasing $(\Delta 226[121] \mathrm{U} / \mathrm{gHb})$ in the OCT group, while GPx decreased $(\Delta 20[13] \mathrm{U} / \mathrm{gHb})$ and MDA increased $(\Delta 72[0.04] \mathrm{nmol} / \mathrm{mL})$ in the CON group. Conclusion: These results suggest that octacosanol may be a beneficial supplement to protect against the poor cholesterol levels and oxidative stress that occurs during taekwondo training.
\end{abstract}

Keywords: sport nutrition, exercise physiology, health

Athletes who participate in weight-division sports such as taekwondo, boxing, judo, and wrestling regularly reduce their body weight severely prior to competition in order to gain a size advantage over the opponent while simultaneously meeting their weight-division requirements. In general, athletes have acute weight loss of 4 to $7 \mathrm{~kg}$ within 3 to 4 days of competition, while longer periods of more gradual weight loss of 15 to $22 \mathrm{~kg}$ occur over 3 to 4 weeks. ${ }^{1,2}$ Although this kind of extreme weight loss can induce metabolic dysfunction, it is repeatedly performed by athletes of weight division sports in order to acquire a more advantageous condition for play. ${ }^{3}$ Furthermore, athletes participating in matches tend to obtain excessive body weights compared with their weight division during the offseason in order to lift heavier weights during training, before rapidly losing that body weight over a short period of time prior to competition. ${ }^{4}$ This rapid weight change with high-intensity exercise training may result in the deterioration of the health and sports performance of these athletes, as it can lead to an abrupt disturbance of metabolism and increased oxidative stress, which can ultimately result in damage to the cells. ${ }^{5}$ This is

(C) 2019 The Authors. Published by Human Kinetics, Inc. This is an Open Access article distributed under the terms of the Creative Commons Attribution License CC BY NC 4.0, which permits unrestricted noncommercial use, distribution, and reproduction in any medium, provided the original work is properly cited, the new use includes a link to the license, and any changes are indicated. See http:// creativecommons.org/licenses/by-nc/4.0. This license does not cover any third-party material which may appear with permission in the article. For commercial use, permission should be requested from Human Kinetics, Inc., through the Copyright Clearance Center (http://www.copyright.com).

S.-H. Lee and J.-K. Lee are with the Dept of Taekwondo, Kosin University, Busan, South Korea. Scott, Pekas, and Park are with the School of Health and Kinesiology, University of Nebraska Omaha, Omaha, NE. Park (Song-youngpark@unomaha. edu) is corresponding author. concerning, as an impairment in lipid metabolism and an increase in free radical production can lead to greater risks of cardiovascular and metabolic disease in these athletes. ${ }^{6,7}$

Octacosanol, a straight-chain, high-molecular-weight, primary aliphatic alcohol minimally contained in the epicuticular wax of various plants may be able to prevent these deteriorations, however, as it has been shown to provide various metabolic and cardiovascular health benefits. ${ }^{8}$ For instance, octacosanol supplementation was shown to prevent an increase in adipose accumulation, serum triglyceride (TG) concentration, and glycogen accumulation, while increasing serum fatty acid content and fatty acid oxidation in experimental models fed a high-fat diet. ${ }^{9-11}$ In humans, octacosanol has been shown to improve cardiovascular function and walking capacity in patients with peripheral artery disease $^{12}$; serum low-density lipoprotein (LDL) and high-density lipoprotein (HDL) concentrations in healthy adults, ${ }^{13}$ and LDL, HDL, total cholesterol, and TG levels in patients with type 2 hypercholesterolemia. ${ }^{14}$ However, the potential beneficial effects of octacosanol intake for improving cholesterol and oxidative stress that occur from sports that require excessive weight change and high-intensity exercise have not been examined. Therefore, the purpose of this study was to examine the effects of octacosanol supplementation during rapid weight loss through caloric restriction with high-intensity exercise training on lipid profiles and markers of oxidative stress in taekwondo players.

\section{Materials and Methods}

\section{Subjects}

All procedures reported in this study were conducted and performed according to the protocols approved by Kosin University's institutional review board (KSU IRB 2017-63-HR) and carried out in 
accordance with the Declaration of Helsinki. This study was also registered with clinicaltrials.gov (NTC03557476). A total of 26 elite male taekwondo athletes (mean age $=18$ [1] y) with at least 3 years of experience in national taekwondo competitions were recruited from Kosin University. Subject characteristics can be seen in Table 1. All participants were healthy and free from cardiovascular, metabolic, and renal diseases, and all participants received complete information about the study design and provided written informed consent. Participants were asked to abstain from caffeinated drinks, alcohol, and antioxidant supplements during the whole period of the study. All participants had no history of chronic use of antioxidants or nonsteroidal anti-inflammatory drugs.

\section{Study Design}

We used a double-blinded, parallel experimental design. Allocation was stratified for years of training ( $>3[n=13$ in OCT group and $\mathrm{n}=13$ in CON group]), and the sequence was generated by a computer-based number. All participants were randomly divided in a 1:1 fashion into 2 groups: (1) a 5\% weight loss with octacosanol intake group (OCT; $\mathrm{n}=13$ ) and (2) a $5 \%$ weight loss with placebo group $(\mathrm{CON} ; \mathrm{n}=13)$ (Figure 1$)$. A power analysis calculation (G*Power 3.1; Universität Kiel, Kiel, Germany) determined a minimum sample size of 26 would allow the observation of a difference of $3 \%$ to $5 \%$ between the groups (OCT vs CON) on the primary study outcome variable of body weight with a power of $80 \%$.

\section{Exercise Protocols}

Participants performed 2 hours of a taekwondo training program at the same time of day over 6 days in a training camp. The intensity of each training sessions was $70 \%$ to $90 \%$ of predicted maximum heart rate. Subjects completed a 10-minute dynamic stretching warm-up before training began. Approximately 1 hour of the taekwondo training program was devoted to technique training and sparring, while the other hour was spent on physical training. The technique training component of the session included basic techniques, simulated fighting techniques, and simulated matches. The physical training component consisted of a circuit training program of interval sprints between various resistance training

Table 1 Physical Characteristics of the Study Participants

\begin{tabular}{|c|c|c|c|c|c|c|c|}
\hline \multirow[b]{2}{*}{ Group } & \multirow[b]{2}{*}{ Age, y } & \multirow[b]{2}{*}{ Height, cm } & \multirow[b]{2}{*}{ Career, mo } & \multicolumn{2}{|c|}{ \%Fat } & \multicolumn{2}{|c|}{ Body weight, kg } \\
\hline & & & & Pre & Post & Pre & Post \\
\hline OCT $(n=13)$ & $18(1)$ & $174.7(1.4)$ & $66(10)$ & $8(2)$ & $5(2)^{*}$ & $66.9(6.7)$ & $62.5(6.9)^{*}$ \\
\hline $\mathrm{CON}(\mathrm{n}=13)$ & $18(1)$ & $173.9(6.3)$ & $69(10)$ & $8(2)$ & $5(2)^{*}$ & $66.7(5.7)$ & $62.3(6.1)^{*}$ \\
\hline
\end{tabular}

Abbreviations: CON, $5 \%$ weight loss with placebo intake; OCT, $5 \%$ weight loss with octacosanol intake. Note: Values are mean (SD).

$* P<.05$, different from pre.

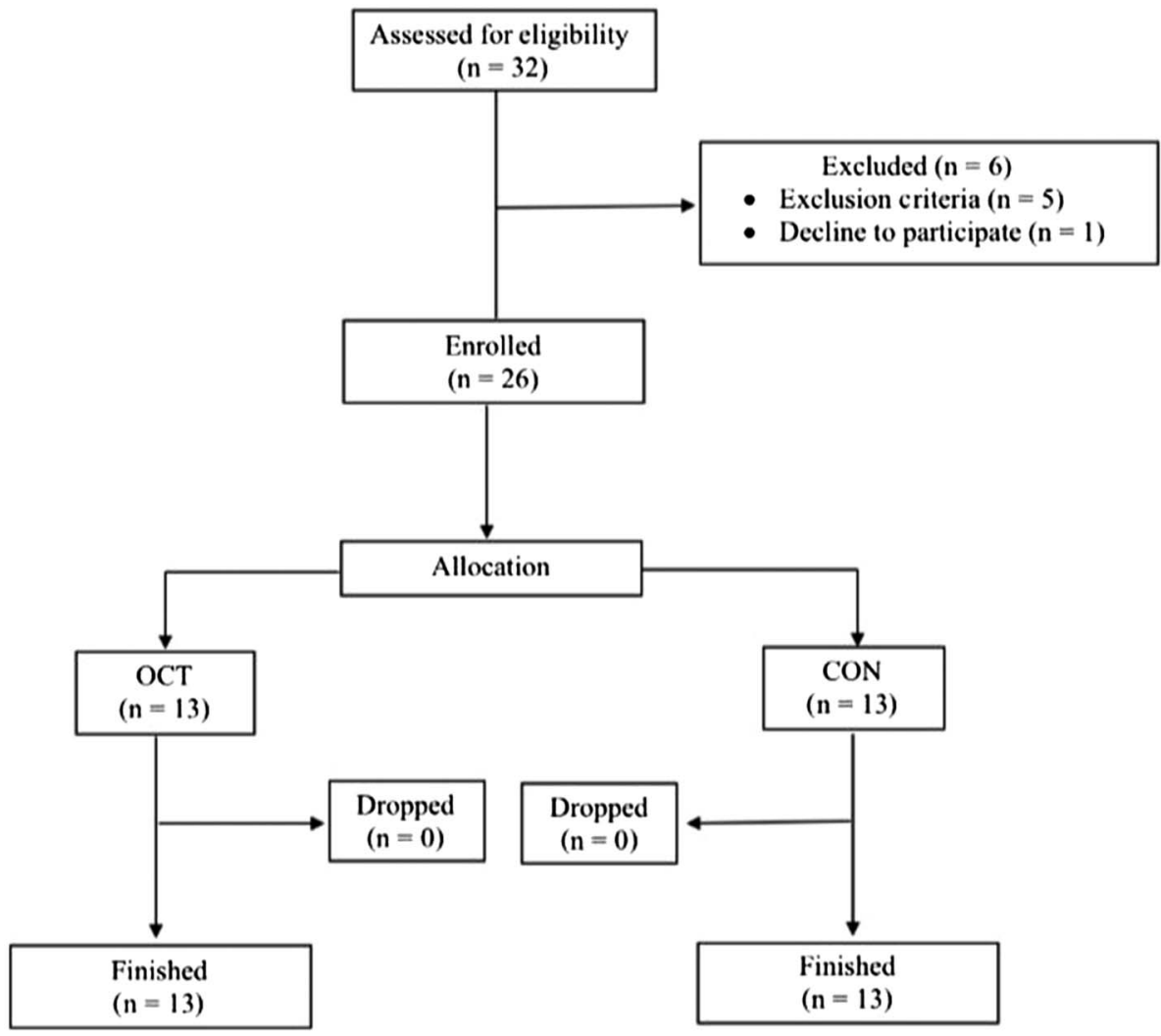

Figure 1 - Diagram for the experimental design of the study. CON indicates placebo-intake group; OCT, octacosanol-intake group. 
Table 2 Taekwondo Training Program

\begin{tabular}{|c|c|c|c|c|c|c|}
\hline Duration, min & & Exe & ises & & Intensity & Frequency, $\mathrm{d} / \mathrm{wk}$ \\
\hline \multirow[t]{2}{*}{10} & Dynamic stretching & & & & - & 6 \\
\hline & Kicks & Punches & Step sparring & Forms & & \\
\hline \multirow[t]{8}{*}{60} & Front kick & Reverse punch & Step forward and step back & Palgwe & $70-90 \% \mathrm{HR}_{\max }$ & 6 \\
\hline & Roundhouse kick & $\mathrm{Jab}$ & 1 step and 2 step & Taegeuk & & \\
\hline & Side kick & Hook punch & & & & \\
\hline & Axe kick & Upper cut & & & & \\
\hline & Back kick & Backfist & & & & \\
\hline & Turn kick & Hammer fist & & & & \\
\hline & Nara kick & & & & & \\
\hline & & Circui & training & & & \\
\hline \multirow[t]{3}{*}{60} & Interval running & Lunges & Squats & Pull-ups & $70-90 \% \mathrm{HR}_{\max }$ & 6 \\
\hline & & Chin-ups & Push-ups & Dips & & \\
\hline & & Crunches & Leg lifts & & & \\
\hline 10 & Static stretching & & & & - & 6 \\
\hline
\end{tabular}

Abbreviation: $\mathrm{HR}_{\max }$, maximum heart rate.

activities. The participants completed a 10-minute cooldown of static stretching at the end of the training session. The training program can be seen in Table 2. Participants wore body protectors during the entirety of the training session, and no injuries were reported. Subjects were allowed to drink water ad libitum during the training sessions.

\section{Dietary Intake}

The participants' lifestyle was highly controlled by researchers during the research period, as the study was conducted at a training camp with food intake and physical activity being supervised by trained instructors. Food logs were obtained during the training period. To lose weight, caloric restriction and exercise were used. Weight was measured every day before and after training sessions from each subject. The nutrient profile was $60 \%$ carbohydrates, $20 \%$ fats, and $20 \%$ protein. Before intervention of caloric restriction, nutritionists calculated the subjects' mean caloric intake for the last 3 days and made a plan for the intake of calories to be progressively reduced by $400 \mathrm{kcal}$ per day, totaling $\sim 2400 \mathrm{kcal}$ over 6 days. Amount of meals were measured by balance (Hanna Instruments, Woonsocket, RI) and provided 3 times per day using a repeated treatment manner.

\section{Intake of Octacosanol}

Two capsules (20 mg each) of $100 \%$ refined octacosanol powder from sugar cane (Swanson, Fargo, ND) were consumed daily by the OCT group for 6 days, 1 capsule 30 minutes after morning and afternoon meals. The intake of octacosanol was followed in a double-blind manner with either a 20-mg capsule or a placebo twice a day. The daily concentration of octacosanol was determined from a previous study. ${ }^{15}$

\section{Collection of Blood}

Before and after the 6 days of this study, blood samples were collected from each subject. Caffeine and any drugs related to vascular function were prohibited 24 hours prior to collection of blood samples. A total of $15 \mathrm{~mL}$ of blood were collected between 9 and 10 AM by syringe (Bom Medrea Co, Ltd, Phnom Penh, Cambodia) after subjects had fasted for 12 hours. The blood samples were immediately centrifuged at $3000 \mathrm{rpm}$ for 10 minutes at $4^{\circ} \mathrm{C}$, and then plasma was collected and stored at $-70^{\circ} \mathrm{C}$ until analysis.

\section{Blood Lipid Profiles}

To examine the levels of plasma lipids, HDL, LDL, total cholesterol, and TG were analyzed in duplicate using a chemistry analyzer (Hitachi 7600-210 and Hitachi 7180, Tokyo, Japan). Plasma samples were mixed with butylated hydroxytoluene and $\mathrm{R} 1$ reagent and reacted for 60 minutes at $45^{\circ} \mathrm{C}$. After the reaction, the mixed samples were centrifuged at $2000 \mathrm{rpm}$ for 10 minutes, and then supernatants were taken. The supernatants were read at $586 \mathrm{~nm}$ by a plate reader.

\section{Redox Balance Measurements}

Plasma levels of superoxide dismutase (SOD) were acquired by SOD Assay Kit-WST (Cayman Chemical, Ann Arbor, MI). Samples were mixed with WST working solution and enzyme working solution, and then the samples were incubated for 20 minutes at $37^{\circ} \mathrm{C}$. The absorbances of the incubated samples were acquired at $450 \mathrm{~nm}$ by plate reader. The SOD activity was determined using a Cobas Mira chemistry analyzer (Roche, Basel, Switzerland). Malondialdehyde (MDA) was measured from BIOXTECHLPO586 kit (Oxis Health Products, Inc, Portland, OR). The plasma samples were incubated with reagent diluent for 5 minutes at room temperature. The incubated samples were stimulated by Ran-Cell total antioxidant control (Randox, Crumlin, United Kingdom) and then analyzed at $340 \mathrm{~nm}$ by plate reader. Plasma levels of glutathione peroxidase (GPx) were acquired by GPx Assay Kit (Cayman Chemical). Samples were incubated for 20 minutes at $37^{\circ} \mathrm{C}$. The absorbances of the incubated samples were acquired at $450 \mathrm{~nm}$ by plate reader. The GPx activity was determined using a Cobas Mira chemistry analyzer (Roche). The plasma samples were incubated with reagent diluent for 5 minutes at room temperature. The incubated samples were stimulated by Ran-Cell total antioxidant control (Randox) and then analyzed at $340 \mathrm{~nm}$ by plate reader.

\section{Anthropometry and Body Composition}

Body composition was assessed between 9 and 10 AM after a 12-hour fast before and after the 6-day training program using an 
8-polar tactile-electrode impedance meter (InBody 720; Biospace, Seoul, South Korea), which simultaneously recorded body weight, fat mass, and fat-free mass. Height was measured with a stadiometer to the nearest $1 \mathrm{~cm}$. Body mass index was calculated as weight in kilograms divided by height in meters squared. ${ }^{16}$

\section{Statistical Analysis}

Data were analyzed using a $2 \times 2$ analysis of variance with repeated measures (group [OCT and $\mathrm{CON}$ ] $\times$ time [before and after $6 \mathrm{~d}$ ]). If a significant main effect or interaction was noted, a paired $t$ test was used for within-group comparisons. Statistical analysis was performed using Statistical Package for the Social Science (SPSS) version 21.0 (IBM SPSS Analytics, Armonk, NY). The values were presented as mean and SE. A value of $P<.05$ was considered as statistically significant.

\section{Results}

\section{Subject Characteristics}

There were not any significant group $\times$ time interactions among groups in age, height, taekwondo experience, or percentage of body fat. Body weight and percentage of body fat following acute weight loss training was significantly lower $(P<.05)$ than pretraining in both groups (Table 1).

\section{Lipid Profiles}

There were significant $(P<.05)$ group $\times$ time differences in HDL, LDL, and TG levels between groups (Figure 2). The OCT group exhibited statistically significant increases in $\mathrm{HDL}(\Delta 10[7] \mathrm{mg} / \mathrm{dL})$ and decreases in LDL $(\Delta 18[5] \mathrm{mg} / \mathrm{dL})$ and TG $(\Delta 80[7] \mathrm{mg} / \mathrm{dL})$ between pretesting and posttesting, while the CON group showed a significant decrease in HDL levels $(\Delta 22[3] \mathrm{mg} / \mathrm{dL})$ after posttesting.

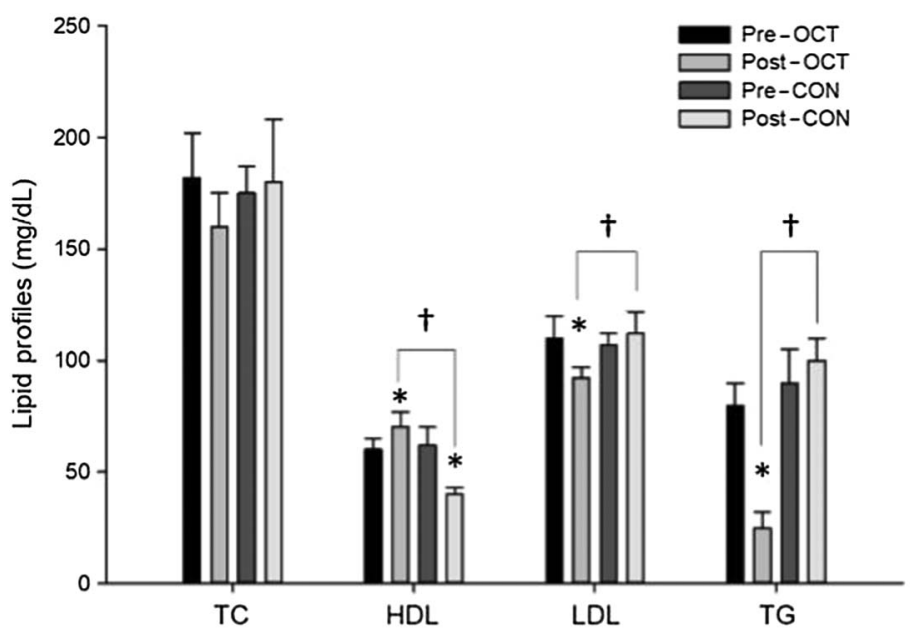

Figure 2 - Changes in lipid profiles before and after 6 days of 5\% weight loss with taekwondo training. HDL increased in the OCT group and decreased in the CON group. In addition, LDL and TG decreased in the OCT group. There were also intergroup differences in HDL, LDL, and TG levels. CON indicates placebo-intake group; HDL, high-density lipoprotein; LDL, low-density lipoprotein; OCT, octacosanol-intake group; TC, total cholesterol; TG, triglyceride. ${ }^{*} P<.05$, different from pre. $\dagger P<.05$, different from CON.

\section{Oxidative Stress}

There were significant $(P<.05)$ group $\times$ time differences between groups in all 3 oxidative stress markers after posttesting. The OCT group had a significant increase in SOD $(\Delta 226[121] \mathrm{U} / \mathrm{gHb})$ after posttesting (Figure $3 \mathrm{~A})$, while the $\mathrm{CON}$ group experienced a significant decrease in GPx $(\Delta 20[13] \mathrm{U} / \mathrm{gHb}$; Figure $3 \mathrm{~B})$ and a significant increase in MDA $(\Delta 72[0.04] \mathrm{nmol} / \mathrm{mL}$; Figure $3 \mathrm{C})$ after posttesting.

\section{Discussion}

After 6 days of a 5\% weight loss through caloric restriction with high-intensity exercise training program, participants who consumed the supplement octacosanol experienced significant improvements in HDL, LDL, and TG levels when compared with the CON group. In addition, SOD, a marker of antioxidant capacity, was significantly increased in the OCT group after 6 days of training, while GPx, an antioxidant enzyme, was significantly reduced in the CON group. MDA, a marker of lipid peroxidation, was also significantly increased in the CON group, but not the OCT group. These results suggest that octacosanol can be used as a beneficial supplement for athletes who go through periods of caloric restriction with high-intensity exercise, as it was able to help protect against the detrimental changes in cholesterol levels and oxidative stress that occur from this kind of training.

The rapid weight loss through caloric restriction with highintensity exercise training seen in taekwondo was shown to decrease levels of HDL and increase levels of LDL and TG, which may eventually increase the risks for both metabolic dysfunction and cardiovascular disease (CVD) in these athletes. However, the OCT group showed an increase in HDL and a reduction in LDL and TG, demonstrating the beneficial effects of octacosanol to reduce the negative changes in lipid levels that occur from this kind of training. HDL is believed to prevent and even remove cholesterol buildup in the arteries, and therefore, its increase would have beneficial effects on cardiovascular health and function, ${ }^{17,18}$ while increased levels of LDL and TG are known to be positively correlated with risks for metabolic and cardiovascular disorders. ${ }^{19,20}$ In fact, both increased HDL and reduced LDL levels have been shown to reduce mortality from CVD. ${ }^{21}$

These observed improvements in the OCT group may be due, in part, to the ability of octacosanol to inhibit the synthesis of cholesterol, as several studies have shown decreases in LDL, total cholesterol, and TG after octacosanol supplementation in both animals and humans. ${ }^{9-12,14}$ However, it seems more likely that octacosanol stimulates the conversion of lipids into energy, as an animal study reported that octacosanol intake resulted in greater serum free fatty acid levels, greater free fatty acid oxidation, and a decreased serum TG concentration when compared with a CON group. ${ }^{9}$ In addition, several studies, along with our own, have shown the ability of octacosanol to elicit an increase in serum HDL, a healthy cholesterol that increases the metabolism of LDL. ${ }^{14,22}$

Another detrimental effect from these periods of rapid weight loss through caloric restriction with high-intensity exercise is an increase in oxidative stress. Free radicals are continuously produced as part of cellular metabolic processes; however, these free radicals are typically neutralized by an endogenous antioxidant defense system consisting of the enzymes SOD and GPx, among others. ${ }^{23}$ During caloric restriction and high-intensity exercise, an 

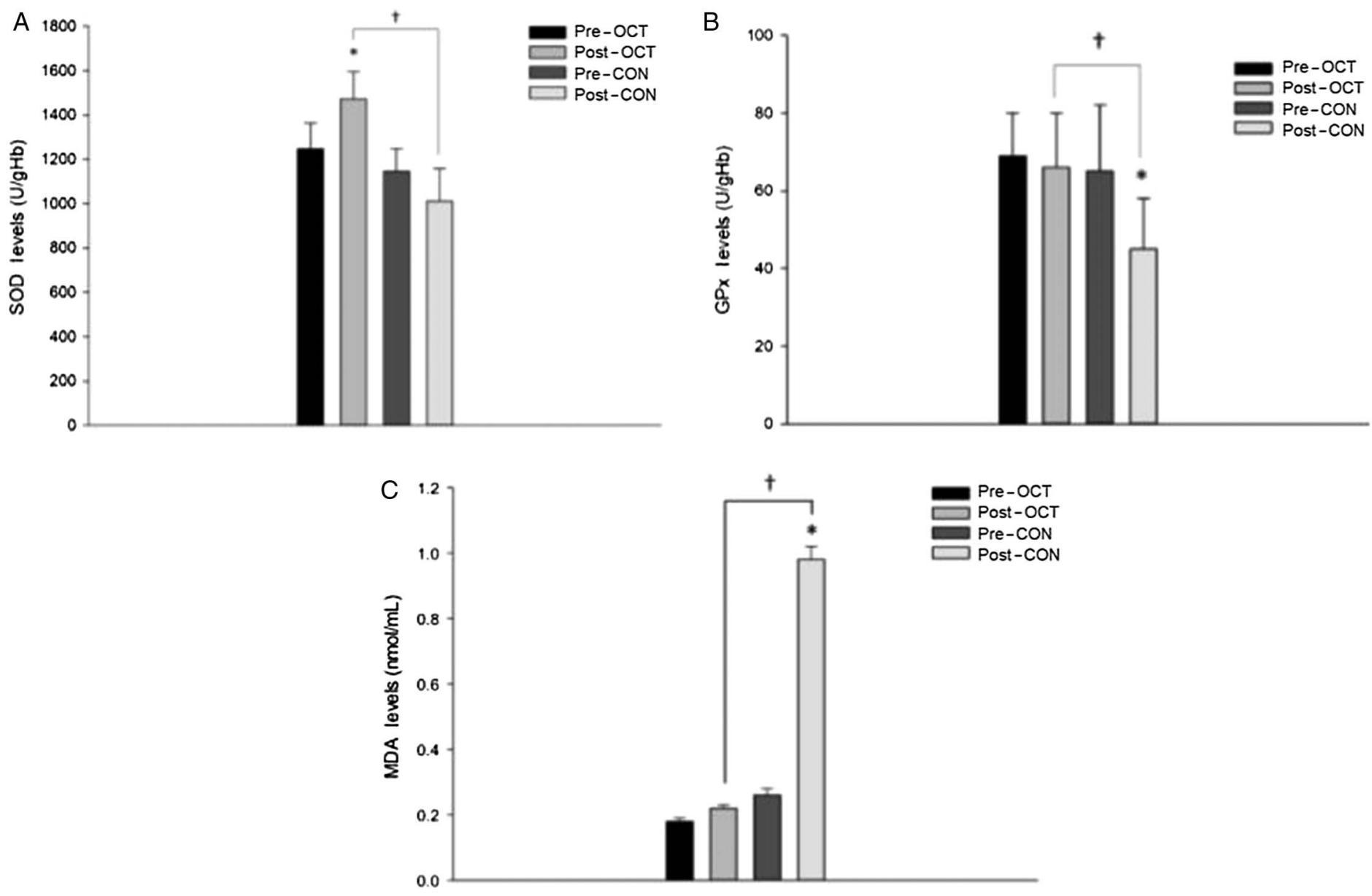

Figure 3 - Changes in SOD, GPx, and MDA levels after 6 days of 5\% weight loss with taekwondo training. (A) SOD levels increased in the OCT group and were higher than in the CON group at posttesting. (B) GPx levels decreased in the CON group and were lower than in the OCT group at posttesting. (C) MDA levels increased in the CON group and were higher than in the OCT group at posttesting. CON indicates placebo-intake group; GPx, glutathione peroxidase; MDA, malondialdehyde; OCT, octacosanol-intake group; SOD, superoxide dismutase. $* P<.05$, different from pre. $† P<.05$, different from CON.

imbalance between free radicals and antioxidants enzymes takes place, leading to oxidative stress. ${ }^{5}$ When this occurs, MDA is produced, a highly reactive chemical and a commonly used marker for oxidative stress, as it induces lipid peroxidation of the cell membrane, ultimately resulting in cell damage. ${ }^{24}$

In this study, MDA was significantly increased in the CON group, while there was no change in the OCT group, demonstrating the effects of this form of sports training to increase lipid peroxidation and the ability of octacosanol to reduce it. Along with its role as a scavenger for oxidative stress, octacosanol also improved antioxidant enzyme levels, as the OCT group had significantly greater SOD levels and the CON group had significantly lower GPx levels. Greater SOD and GPx levels, as seen in the OCT group, would be beneficial, as SOD eliminates harmful free oxygen radicals ${ }^{25}$ and GPx reduces the damaging effects of lipid peroxidation. ${ }^{26}$ These improvements are believed to be due to the antioxidant effects of octacosanol, as one study found strong antiradical properties of octacosanol $(96.42 \%$ and $90.35 \%$ antiradical activity from the DPPH and ABTS radical scavenging assays, respectively), ${ }^{27}$ while another study demonstrated the ability of octacosanol to decrease oxidative stress markers in an animal model. ${ }^{28}$
This form of sports training may also lead to an increase in systemic inflammation, as inflammatory markers, such as C-reactive protein and interleukin-6, have been shown to be correlated with elevated lipid profiles and oxidative stress. ${ }^{29}$ An increase in these markers have also been linked to increased risks for CVD. ${ }^{30}$ Although not measured in this study, we believe this warrants further research, as octacosanol supplementation was shown to reduce both oxidative stress and LDL cholesterol levels in the OCT group. This suggests that it may also potentially reduce levels of inflammation that likely increase with this form of training as well.

Since taekwondo is a weight division sport, the participants of this sport repeatedly go through periods of rapid weight change with high-intensity exercise training in order to train at heavier body weights and then compete in lighter weight divisions. This results in poor lipid profiles and high levels of oxidative stress. Repeated exposure to this form of severe weight change with highintensity training can result in impaired physiological function, which may eventually increase risks of metabolic dysfunction and CVD in these athletes. Therefore, it is important to find a nutritional treatment, such as octacosanol supplementation, in order to protect against the negative effects of this kind of sports training. 


\section{Practical Applications}

These findings reveal that rapid weight loss paired with highintensity exercise leads to poor lipid profiles and elevated levels of oxidative stress in taekwondo athletes. Chronic training in this manner may result in poor metabolic and cardiovascular health in these athletes and may interfere with sports performance. This study has shown that octacosanol supplementation reduced negative cholesterol and oxidative stress levels during taekwondo training, making it a potential therapeutic intervention in order to improve physiological function and prevent metabolic dysfunction in these athletes. Future research on octacosanol should examine larger sample sizes in various sports, as well as markers for inflammation.

\section{Conclusion}

As we have demonstrated in this study, octacosanol supplementation during rapid weight loss through caloric restriction with highintensity exercise training improved lipid profiles and the balance between antioxidant enzymes and oxidative stress markers in taekwondo athletes. These findings suggest that octacosanol is a useful supplement for counteracting the potential negative effects of this form of training on health and performance in these kinds of athletes.

\section{Acknowledgments}

The authors are grateful to the participants. There was no funding source for this study. No conflicts of interest, financial or otherwise, are declared by the authors. Please contact author for data requests. Conception and design of the experiments: S.-H.L., J.-K.L., and S.-Y.P. Collection, assembly, analysis, and interpretation of data: S.-H.L., S.D.S., E.J.P., J.-K.L., and S.-Y.P. Drafting the article or revising it critically for important intellectual content: S.-H.L., S.D.S., E.J.P., J.-K.L., and S.-Y.P. This study was registered with clinicaltrials.gov (NTC03557476).

\section{References}

1. Brownell KD, Steen SN, Wilmore JH. Weight regulation practices in athletes: analysis of metabolic and health effects. Med Sci Sports Exerc. 1987;19:546-556. PubMed ID: 3323766 doi:10.1249/ 00005768-198712000-00002

2. Zambraski EJ, Tipton CM, Tcheng TK, Jordon HR, Vailas AC, Callahan AK. Iowa wrestling study: changes in the urinary profiles of wrestlers prior to and after competition. Med Sci Sports. 1975; 7:217-220. PubMed ID: 1625

3. Fogelholm GM, Koskinen R, Laakso J, Rankinen T, Ruokonen I. Gradual and rapid weight loss: effects on nutrition and performance in male athletes. Med Sci Sports Exerc. 1993;25:371-377. PubMed ID: 8455453 doi:10.1249/00005768-199303000-00012

4. Steen SN, Brownell KD. Patterns of weight loss and regain in wrestlers: has the tradition changed? Med Sci Sports Exerc. 1990; 22:762-768. PubMed ID: 2287253 doi:10.1249/00005768199012000-00005

5. Alessio HM. Exercise-induced oxidative stress. Med Sci Sports Exerc. 1993;25:218-224. PubMed ID: 8383786 doi:10.1249/ 00005768-199302000-00010

6. Booth FW, Roberts CK. Linking performance and chronic disease risk: indices of physical performance are surrogates for health. $\mathrm{Br} J$
Sports Med. 2008;42:950-952. PubMed ID: 18838401 doi:10.1136/ bjsm.2008.052589

7. Mahjoub S, Masrour-Roudsari J. Role of oxidative stress in pathogenesis of metabolic syndrome. Caspian J Intern Med. 2012;3: 386-396. PubMed ID: 26557292

8. Taylor JC, Rapport L, Lockwood GB. Octacosanol in human health. Nutrition. 2003;19:192-195. PubMed ID: 12591561 doi:10.1016/ S0899-9007(02)00869-9

9. Kato S, Karino K, Hasegawa S, et al. Octacosanol affects lipid metabolism in rats fed on a high-fat diet. Br J Nutr. 1995;73: 433-441. PubMed ID: 7766566 doi:10.1079/BJN19950045

10. Shimura S, Hasegawa T, Takano S, Suzuki T. Studies on the effect of octacosanol on motor endurance in mice. Nutr Rep Int. 1987;36: 1029-1038.

11. Arruzazabala ML, Carbajal D, Mas R, Molina V, Valdes S, Laguna A. Cholesterol-lowering effects of policosanol in rabbits. Biol Res. 1994;27:205-208. PubMed ID: 8728832

12. Castano G, Mas Ferreiro R, Fernandez L, Gámez R, Illnait J, Fernández C. A long-term study of policosanol in the treatment of intermittent claudication. Angiology. 2001;52:115-125. PubMed ID: 11228084 doi:10.1177/000331970105200205

13. Hernandez F, Illnait J, Mas R, et al. Effect of policosanol on serumlipids and lipoproteins in healthy-volunteers. Curr Ther Res Clin Exp. 1992;51:568-575.

14. Mas R, Castano G, Illnait J, et al. Effects of policosanol in patients with type II hypercholesterolemia and additional coronary risk factors. Clin Pharmacol Ther. 1999;65:439-447. PubMed ID: 10223782 doi:10.1016/S0009-9236(99)70139-6

15. Arruzazabala ML, Molina V, Mas R, et al. Antiplatelet effects of policosanol (20 and $40 \mathrm{mg} /$ day) in healthy volunteers and dyslipidaemic patients. Clin Exp Pharmacol Physiol. 2002;29:891-897. PubMed ID: 12207568 doi:10.1046/j.1440-1681.2002.03746.x

16. Wong A, Figueroa A, Son WM, Chernykh O, Park SY. The effects of stair climbing on arterial stiffness, blood pressure, and leg strength in postmenopausal women with stage 2 hypertension. Menopause. 2018;25(7):731-737. PubMed ID: 29438269 doi:10.1097/GME. 0000000000001072

17. Bezafibrate Infarction Prevention Study. Secondary prevention by raising HDL cholesterol and reducing triglycerides in patients with coronary artery disease. Circulation. 2000;102:21-27. doi:10.1161/ 01.CIR.102.1.21.

18. Pearson TA, Bulkley BH, Achuff SC, Kwiterovich PO, Gordis L. The association of low levels of HDL cholesterol and arteriographically defined coronary artery disease. Am J Epidemiol. 1979;109:285-295. PubMed ID: 222134 doi:10.1093/oxfordjournals.aje.a112682

19. Castelli WP, Cooper GR, Doyle JT, et al. Distribution of triglyceride and total, LDL and HDL cholesterol in several populations: a cooperative lipoprotein phenotyping study. J Chronic Dis. 1977; 30:147-169. PubMed ID: 191465 doi:10.1016/0021-9681(77) 90082-0

20. Manninen V, Tenkanen L, Koskinen P, et al. Joint effects of serum triglyceride and LDL cholesterol and HDL cholesterol concentrations on coronary heart disease risk in the Helsinki Heart Study. Implications for treatment. Circulation. 1992;85:37-45. PubMed ID: 1728471 doi:10.1161/01.CIR.85.1.37

21. Gotto AM, Gorry GA, Thompson JR, et al. Relationship between plasma lipid concentrations and coronary artery disease in 496 patients. Circulation. 1977;56:875-883. PubMed ID: 912850 doi:10.1161/01.CIR.56.5.875

22. Gamez R, Maz R, Arruzazabala ML, Mendoza S, Castaño G. Effects of concurrent therapy with policosanol and omega-3 fatty acids on lipid profile and platelet aggregation in rabbits. Drugs $R$ D. 2005; 
6:11-19. PubMed ID: 15806713 doi:10.2165/00126839-20050601000002

23. Urso ML, Clarkson PM. Oxidative stress, exercise, and antioxidant supplementation. Toxicology. 2003;189:41-54. PubMed ID: 12821281 doi:10.1016/S0300-483X(03)00151-3

24. Jacob RA, Burri BJ. Oxidative damage and defense. Am J Clin Nutr. 1996;63:985S-990S. PubMed ID: 8644698 doi:10.1093/ajcn/63. 6.985

25. Nishikawa T, Edelstein D, Du XL, et al. Normalizing mitochondrial superoxide production blocks three pathways of hyperglycaemic damage. Nature. 2000;404:787-790. PubMed ID: 10783895 doi:10. 1038/35008121

26. Thomas JP, Maiorino M, Ursini F, Girotti AW. Protective action of phospholipid hydroperoxide glutathione peroxidase against membranedamaging lipid peroxidation. In situ reduction of phospholipid and cholesterol hydroperoxides. J Biol Chem. 1990;265:454-461. PubMed ID: 2294113
27. Harrabi S, Ferchichi A, Bacheli A, Fellah H. Policosanol composition, antioxidant and anti-arthritic activities of milk thistle (Silybium marianum L.) oil at different seed maturity stages. Lipids Health Dis. 2018;17:82. PubMed ID: 29661192 doi:10.1186/s12944-018-0682-Z

28. Ohta Y, Ohashi K, Matsura T, Tokunaga K, Kitagawa A, Yamada K. Octacosanol attenuates disrupted hepatic reactive oxygen species metabolism associated with acute liver injury progression in rats intoxicated with carbon tetrachloride. J Clin Biochem Nutr. 2008; 42:118-125. PubMed ID: 18385828 doi:10.3164/jcbn.2008017

29. Ridker PM, Rifai N, Rose L, Buring JE, Cook NR. Comparison of $\mathrm{C}$-reactive protein and low-density lipoprotein cholesterol levels in the prediction of first cardiovascular events. N Engl J Med. 2002;347: 1557-1565. PubMed ID: 12432042 doi:10.1056/NEJMoa021993

30. Ridker PM, Hennekens CH, Buring JE, Rifai N. C-reactive protein and other markers of inflammation in the prediction of cardiovascular disease in women. $N$ Engl J Med. 2000;342:836-843. PubMed ID: 10733371 doi:10.1056/NEJM200003233421202 